\title{
Dimensional Stability and Microstructure Evolution in Irradiated Systems with Complex Kinetics
}

T. Diaz de la Rubia, M.J. Caturla, M.J. Fluss

\section{October 11, 1999}

Lawrence

Livermore

National

Laboratory 


\section{DISCLAIMER}

This document was prepared as an account of work sponsored by an agency of the United States Government. Neither the United States Government nor the University of California nor any of their employecs, makes any warranty, express or implied, or assumes any legal liability or responsibility for the accuracy, completeness, or usefulness of any information, apparatus, product, or process disclosed, or represents that its use would not infringe privately owned rights. Reference herein to any specific commercial product, process, or service by trade name, trademark, manufacturer, or otherwise, does not necessarily constitute or imply its endorsement, recommendation, or favoring by the United States Government or the University of California. The views and opinions of authors expressed herein do not necessarily state or reflect those of the United States Government or the University of California, and shall not be used for advertising or product endorsement purposes.

Work performed under the auspices of the U.S. Department of Energy by the University of California Lawrence Livermore National Laboratory under Contract W-7405-Eng-48.

This report has been reproduced directly from the best available copy.

Available to DOE and DOE contractors from the

Office of Scientific and Technical Information

P.O. Box 62, Oak Ridge, TN 37831

Prices available from (423) 576-8401

http://apollo.osti.gov/bridge/

Available to the public from the

National Technical Information Service

U.S. Department of Commerce 5285 Port Royal Rd., Springfield, VA 22161 http://www.ntis.gov/

OR

Lawrence Livermore National Laboratory Technical Information Department's Digital Library http://www.llnl.gov/tid/Library.html 


\title{
Dimensional Stability and Microstructure Evolution in Irradiated Systems with Complex Kinetics
}

\author{
Tomas Diaz de la Rubia, Maria Jose Caturla, and Michael J. Fluss \\ Chemistry and Materials Science Directorate \\ Lawrence Livermore National Laboratory \\ October 11, 1999
}

\begin{abstract}
We use a combination of molecular dynamics and kinetic Monte Carlo simulations to explore the role of temperature and dose rate on damage accumulation in a model system with complex kinetics. We describe the accumulation of He-vacancy ( $\mathrm{HeV})$ complexes as well as vacancy and interstitial clusters as a function of irradiation temperature, dose, and dose rate. We show that nucleation of stable $\mathrm{HeV}$ complexes (voids and bubbles) at low temperature and flux takes place at extremely low doses. We also describe the effect of temperature on the $\mathrm{HeV}$ complex size distribution and show that growth beyond a critical nucleation size is not possible in this system at temperatures above 300 $\mathrm{K}$ for dose rates smaller than $10^{-8} \mathrm{dpa} / \mathrm{s}$. We further demonstrate that a temperature shift of $25 \mathrm{~K}$ per decade of flux scales the dose rate dependence of He-vacancy complex (voids and bubbles) accumulation when irradiation is carried out to low doses $(0.03-0.06 \mathrm{dpa})$ at temperatures between $150 \mathrm{~K}$ and $300 \mathrm{~K}$ and dose rates of $10^{-6}, 10^{-7}, 10^{-8}$, and $10^{-9} \mathrm{dpa} / \mathrm{s}$. The results provide an atomistic description of microstructure evolution including void nucleation and the early stages of growth, and should be useful in designing and interpreting accelerated aging experiments.
\end{abstract}

\section{INTRODUCTION}

A material undergoing irradiation with energetic particles (e.g. neutrons, ions, or alpha-decay processes) evolves in time. This evolution is a result of the interaction among the produced defects and the pre-existing microstructure, and is a complex function of irradiation temperature, dose (time), and dose rate (flux). The multiple microstructural features that develop such as self-interstitial (I) and vacancy loops, Hevacancy (HeV) complexes (voids, bubbles, etc.), and other defect clusters each follow their own complex kinetics. It is therefore interesting to investigate whether scaling laws may be formulated that relate the rate of damage accumulation to the irradiation temperature and flux. Is a particular feature of the microstructural state reached after a certain dose independent of dose rate, and if not, what are the scaling laws that allow comparisons to be made of experiments under different kinetic conditions?

Experiments that use accelerated (increased flux) irradiation as a means of probing microstructure evolution in lower flux systems require robust theoretical and computational models from which extrapolations can be safely made. In this report atomistic simulation tools are used to show that in some instances scaling laws exist, and that therefore accelerated irradiation experiments are a reasonable way of investigating 
microstructure evolution in slow damaging systems. We argue that our multiscale modeling approach provides a reliable tool that should aid in experiment design.

We present results for simulations of damage accumulation in a model system that mimics an FCC metal with low melting point $\left(\mathrm{T}_{\mathrm{m}}=950 \mathrm{~K}\right)$, high atomic number $(\mathrm{Z}=196)$, and a commensurate self diffusion coefficient of $\mathrm{Q}=1.38 \mathrm{eV}$. The system self damages through a combination of $30 \mathrm{keV}$ self-recoil displacement cascades and $1 \mathrm{MeV} \mathrm{He}$ recoils. The calculations are carried out at temperatures from $180 \mathrm{~K}$ to $400 \mathrm{~K}$ (i.e. from annealing stage III past stage V). We explore the effect of dose rate by varying it between $10^{-6} \mathrm{dpa} / \mathrm{s}$ and $10^{-9} \mathrm{dpa} / \mathrm{s}$. The calculations are carried out to a maximum dose of $0.06 \mathrm{dpa}$. More details of the simulated system are given below. The results show that the accumulation of intra-cascade vacancy clusters (Frank sessile loops and stacking fault tetrahedra) and I dislocation loops (both sessile and glissile) at temperatures where these are stable can be simply scaled with the dose rate. On the other hand, features such as small voids and bubbles ( $\mathrm{HeV}$ complexes) form as a result of diffusion at all temperatures studied. We demonstrate that at low dose rates and temperatures $\mathrm{HeV}$ complexes nucleate and grow above the critical size even at extremely low doses. In addition, we show that a $25 \mathrm{~K}$ temperature shift per decade of increase in dose rate scales the dose rate dependence of $\mathrm{HeV}$ complex accumulation appropriately. Given identical sets of experimental conditions (including initial microstructure state), these results show that in this regime of flux and temperature accelerated aging experiments coupled with multiscale modeling should be an appropriate tool to investigate aging in stockpile materials.

\section{METHOD}

Our calculations start with a molecular dynamics (MD) simulation of defect production from displacement cascades [1]. The kinetics of damage accumulation is probed with a kinetic Monte Carlo (KMC) model [2]. Both these types of calculations have been described extensively in recent publications and will not be detailed here. Suffice it to say that this approach links time scales from picoseconds to months and length scales from nanometers to microns. The approach has been used successfully [3] to investigate damage accumulation in irradiated $\mathrm{Cu}$ and $\mathrm{Fe}$ to doses up to $10^{-2} \mathrm{dpa}$, where dpa is the standard unit of damage as defined in the ASTM standards [4].

While MD provides an exquisitely detailed picture of defect production in cascades, the issue of damage accumulation and its dependence on temperature and flux cannot be answered from looking at these results alone. For this purpose one requires a simulation method that can describe the long time scale associated with diffusion of all the defect species produced in a cascade. Such a method must be able to track the fate of all possible clusters that may ensue, from the smallest units consisting of two defects, to the largest possible dislocation loops, cavities, etc. Here, we have chosen to use the KMC method. While computationally demanding, $\mathrm{KMC}$ is very powerful in that it retains the atomistic character of the problem while at the same time properly treating its stochastic nature. No mean field approximations are required and damage accumulation is properly treated as a non-homogeneous process in both space and time. At present, the drawback 
is that calculations can only be carried out to relatively low irradiation times corresponding to accumulated damage doses of the order of $10^{-2}$ to 1 dpa depending on dose rate.

Our KMC simulation code tracks the kinetics of vacancies, self interstitial atoms (SIAs), impurities, and their clusters. Kinetic input to the code consists of the migration energies of defects and impurities and their binding energies to clusters of all sizes. The primary defect distributions are taken from the MD and TRIM calculations described below. The $\mathrm{KMC}$ simulation evolves as follows:

(i) A displacement cascade from a MD simulation is chosen from a pre-existing library together with the damage from a TRIM trajectory of a He atom. The coordinates of all the defects are translated into the KMC box and used as input

(ii) The KMC simulation proceeds by moving all the defects at a specified temperature for a time given by the irradiation flux. The defect that executes a particular diffusion jump at a given time is chosen in a Monte Carlo fashion with a probability based on its diffusivity or binding energy. Time is calculated from the defect diffusivities as described in detailed elsewhere [3].

(iii) At the specified time given by the flux another pair of MD/TRIM cascades are introduced at random into the KMC box.

(iv) The KMC simulation proceeds and defects migrate, interact, recombine, etc.

(v) This loop is carried out until the total desired dose (number of cascades) has been accumulated.

The investigations are carried out in a model system with the following characteristics:

(i) An embedded atom method interatomic potential appropriate to $\mathrm{Au}$ is used in MD simulations of damage production in displacement cascades.

(ii) Damage from cascades is produced by $30 \mathrm{keV} \mathrm{Au}$ recoils in $\mathrm{Au}$ in computational boxes with 1 million atoms.

(iii) Damage from alpha particles is calculated with the TRIM code for $1 \mathrm{MeV} \mathrm{He}$ in Au.

(iv) In the $\mathrm{KMC}$ simulations, for every Au cascade introduced into the box a cascade from the alpha particle irradiation is also introduced.

(v) The melting point of the model system is $\mathrm{T}_{\mathrm{m}}=950 \mathrm{~K}$

(vi) The vacancy activation energy for migration is $\mathrm{E}_{\mathrm{m}}(\mathrm{V})=0.68 \mathrm{eV}$.

(vii) The coefficient of self diffusion is $\mathrm{Q}=1.38 \mathrm{eV}$.

(viii) The activation energy for interstitial He migration is $\mathrm{E}_{\mathrm{He}}=0.1 \mathrm{eV}$;

(ix) The activation energy for $\mathrm{HeV}$ migration is $\mathrm{E}_{\mathrm{HeV}}=0.68 \mathrm{eV}$;

(x) The activation energy for self interstitial atom (SIA) diffusion is $\mathrm{E}_{\mathrm{m}}(\mathrm{I})=0.1 \mathrm{eV}$. The SIA migrates in 3D. The same is true for SIA clusters of size $\mathrm{N}_{\mathrm{l}}=2,3$.

(xi) SIA clusters with $\mathrm{N}_{\mathrm{r}}>3$ migrate in $1 \mathrm{D}$ with $\mathrm{E}_{\mathrm{a}}=0.1 \mathrm{eV}$. SIA clusters larger than 30 are sessile.

(xii) Small vacancy clusters are bound with binding energies calculated by MD. Large vacancy clusters dissociate according to the self diffusion energy given above. An interpolation function is used for intermediate size clusters. Details have been given elsewhere [5]. 
(xiii) SIA clusters do not dissociate at any of the temperatures studied.

(xiv) $\mathrm{HeV}$ cluster binding energies were obtained from published data in $\mathrm{Ni}[6]$ and scaled by a melting point $\mathrm{T}_{\mathrm{m}}=950 \mathrm{~K}$

(xv) All defect reactions are diffusion limited

(xvi) No impurities or solute atoms are considered

(xvii) The grain size of the crystal is $1 \mu \mathrm{m}$. Grain boundaries are sinks for defects.

\section{RESULTS}

MD simulations have been used extensively to study displacement cascades and few details will be provided here. For more information the reader is referred to the recent review article by Averback and Diaz de la Rubia [1]. The simulations are carried out in the ASCI Blue CTR supercomputer with the MDCASK code that was developed at LLNL by Caturla and Chan [7]. The code can solve the classical equations of motion of an ensemble of atoms at a rate of $1 \times 10^{-7}$ sec/atom/time-step on 220 nodes ( 44 processors/node). A prototypical result for a $30 \mathrm{keV} \mathrm{Au}$ cascade in $\mathrm{Au}$ is shown in Figure 1. After the displacement cascade cools, defects are left behind in the form of vacancy and SIA clusters. Also shown in the figure are contour plots of the stress state of

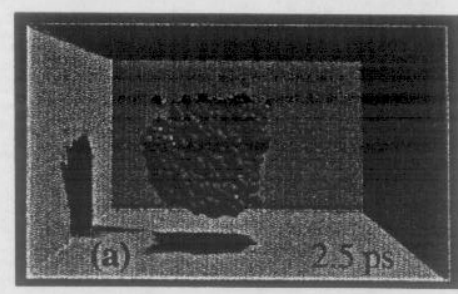

(Maximum defects)

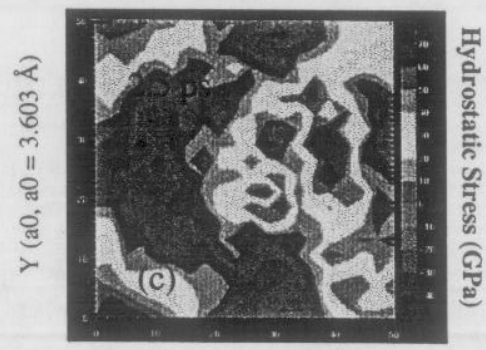

$\mathrm{X}(\mathrm{a} 0, \mathrm{a} 0=3.603 \AA)$

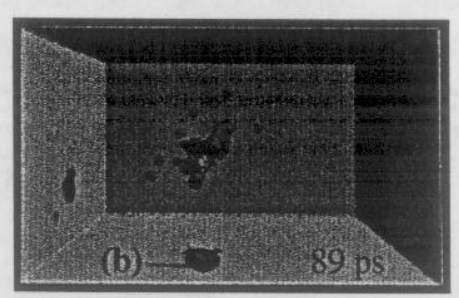

(Final configuration)

면

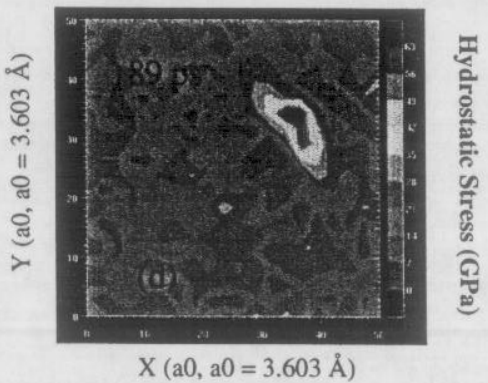

Figure 1. $30 \mathrm{keV}$ Displacement casaced in Au. V clusters (green) and I loops (red) form as a result of the displacment cascade on a time scale of $100 \mathrm{ps}$. The I clusters are highly mobile. The countour plots show the hydrostatic stress in a slab across the center of the cascade. Red is compressive and blue tensile stress. a slab of the crystal during the evolution of the cascade. Note that a region of large tensile stress (blue in the figure) is established early on in the core of the cascade. This low density region is equivalent to a molten core and lasts for several tens of lattice vibration periods. The periphery of this core is generally in a state of compressive stress. After the energy deposited in the cascade core dissipates and the region cools, vacancies appear where the molten core had been, while SIA clusters are evident in the

periphery. These simulations also show that the total number of defects produced per cascade is only $30 \%$ [1] of that predicted by the simple ballistic model associated with the ASTM standard measure [4], the so-called dpa. Also important to emphasize is the fact that defects produced by displacement cascades in high Z, low melting point metals such as Au are in clusters. They are not produced as isolated Frenkel Pairs. On the other 
hand, defects produced by an $\mathrm{MeV} \mathrm{He}$ atom are mostly produced in the form of uncorrelated Frenkel pairs with a few low energy cascades taking place at the end of range. We use the TRIM code to quantify defect production along the trajectory of the $\mathrm{He}$ atom, but no details are provided here.

For the system described above, we have calculated the rates of vacancy and SIA cluster and $\mathrm{HeV}$ complex accumulation as a function of time for different temperatures and fluxes. Figure 2 shows the rate of (a) vacancy and (b) SIA cluster accumulation at several temperatures for a dose rate of $10^{-8} \mathrm{dpa} / \mathrm{s}$. The total number of vacancy and interstitial clusters decreases with increasing temperature. At $225 \mathrm{~K}$ small vacancy clusters start to dissociate and by $350 \mathrm{~K}$ none survive. While the vacancy cluster number density decreases because of dissolution of small clusters as the temperature increases, the interstitial cluster concentration decreases because of recombination with mobile
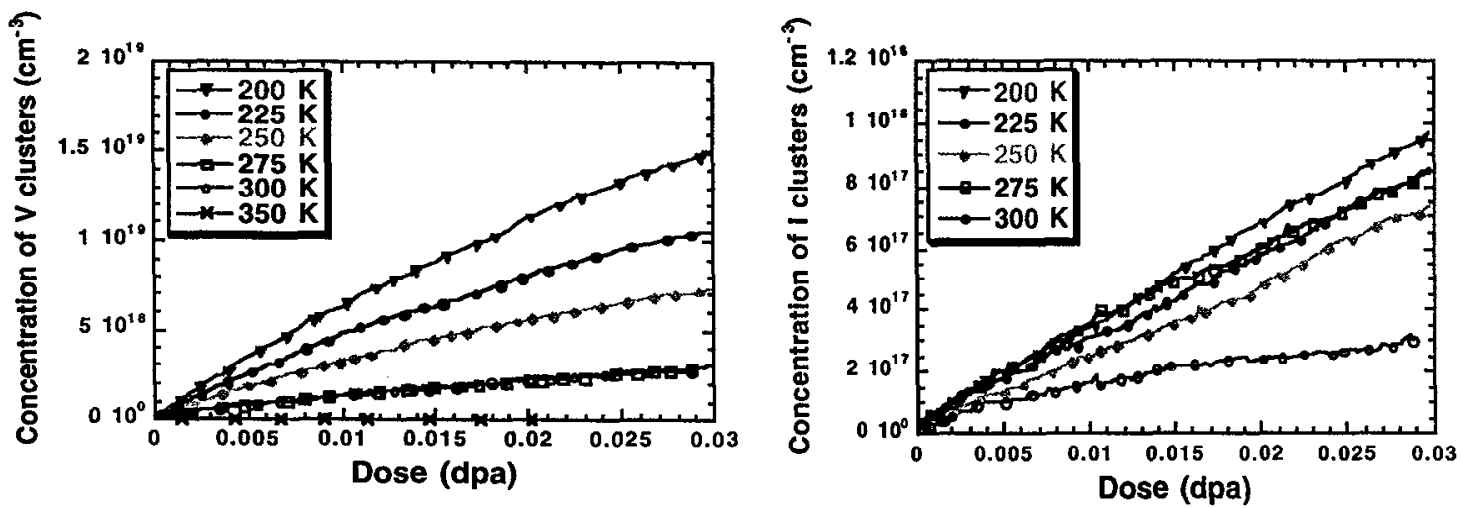

Figure 2. Rate of (a) vacancy cluster and (b) I cluster accumulation with dose at severa temperatures for irradiation at $10^{-8} \mathrm{dpa} / \mathrm{s}$

vacancies and migration to sinks. Also, the total instantaneous concentration of vacancy clusters is much larger than that of SIA clusters because the latter are able to quickly diffuse to sinks.

Figure 3 shows the rate of accumulation of $\mathrm{HeV}$ clusters. Because He stabilizes small

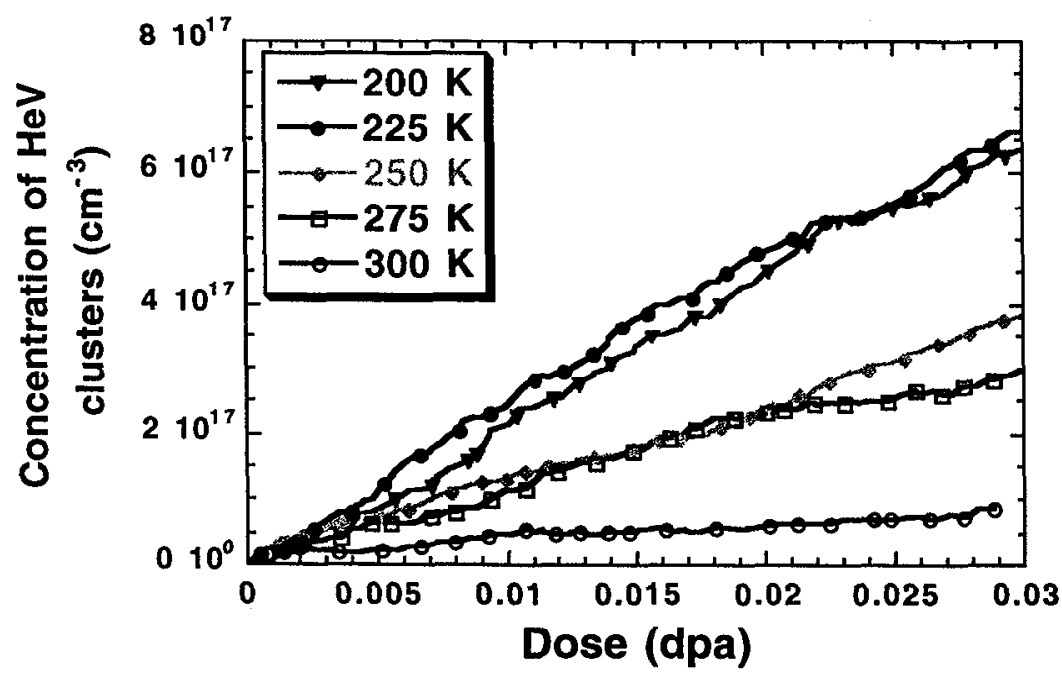

vacancy clusters, the decrease in $\mathrm{HeV}$ cluster concentration with dose comes at a higher temperature, $250 \mathrm{~K}$, than in the case of pure vacancy clusters. From the results we have observed that at $200 \mathrm{~K}$ the vast majority of the clusters contain at most one $\mathrm{He}$ atom and the ratio of $\mathrm{He}$ to vacancy content is small. At $225 \mathrm{~K}$, the

Figure 3. Concentration of $\mathrm{HeV}$ bubbles and voids as a function of dose for irradiation at several temperatures at a dose rate of $10^{-8} \mathrm{dpa} / \mathrm{s}$ 
distribution shifts slightly toward larger sizes and larger He/V ratios. The effect is most pronounced at $275 \mathrm{~K}$ where clusters with as many as $5 \mathrm{He}$ atoms and $\mathrm{He} / \mathrm{V}$ ratios of 0.5 are found in the simulations. These may be thought of as bubble embryos and are thermally stable at this temperature. At $300 \mathrm{~K}$, small $\mathrm{HeV}$ clusters are rather unstable and nucleation of bubbles becomes difficult thus greatly reducing the rate of cluster accumulation with dose.

This ripening of the vacancy and $\mathrm{HeV}$ cluster populations with temperature can also be observed in Figure 4. Here, the cluster density is plotted versus size for the same conditions as in Figs. 2 and 3. Small vacancy clusters dissolve at $\mathrm{T}>225 \mathrm{~K}$ and this gives rise to a decrease in number density and an increase in average size of the cluster population. For $\mathrm{HeV}$ complexes the effect is very clear at $275 \mathrm{~K}$ where small clusters dissolve and the population shifts significantly to larger sizes as seen in Fig. 4b.

(a)

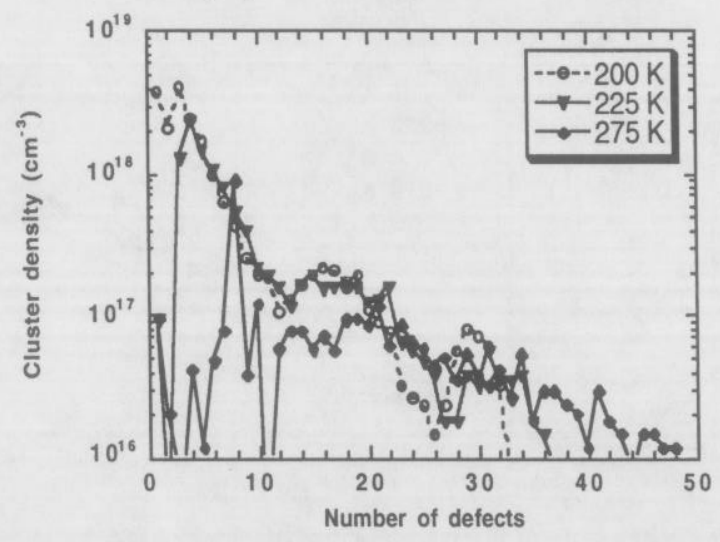

(b)

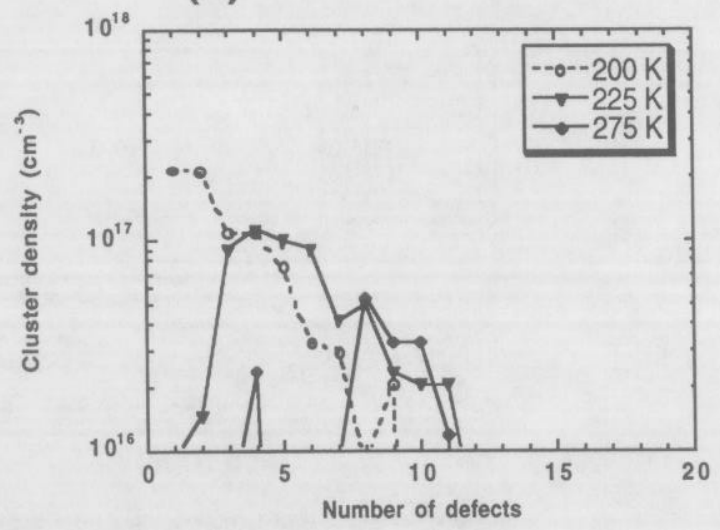

Figure 4. Evolution of (a) the vacancy cluster and (b) the HeV cluster populations with irradiation temperature at $10^{-8} \mathrm{dpa} / \mathrm{s}$

The effect of dose rate on $\mathrm{HeV}$ complex nucleation and early growth can be seen in Figure 5. Here, the population of $\mathrm{HeV}$ complexes is plotted after $0.012 \mathrm{dpa}$ at $\mathrm{T}=225 \mathrm{~K}$

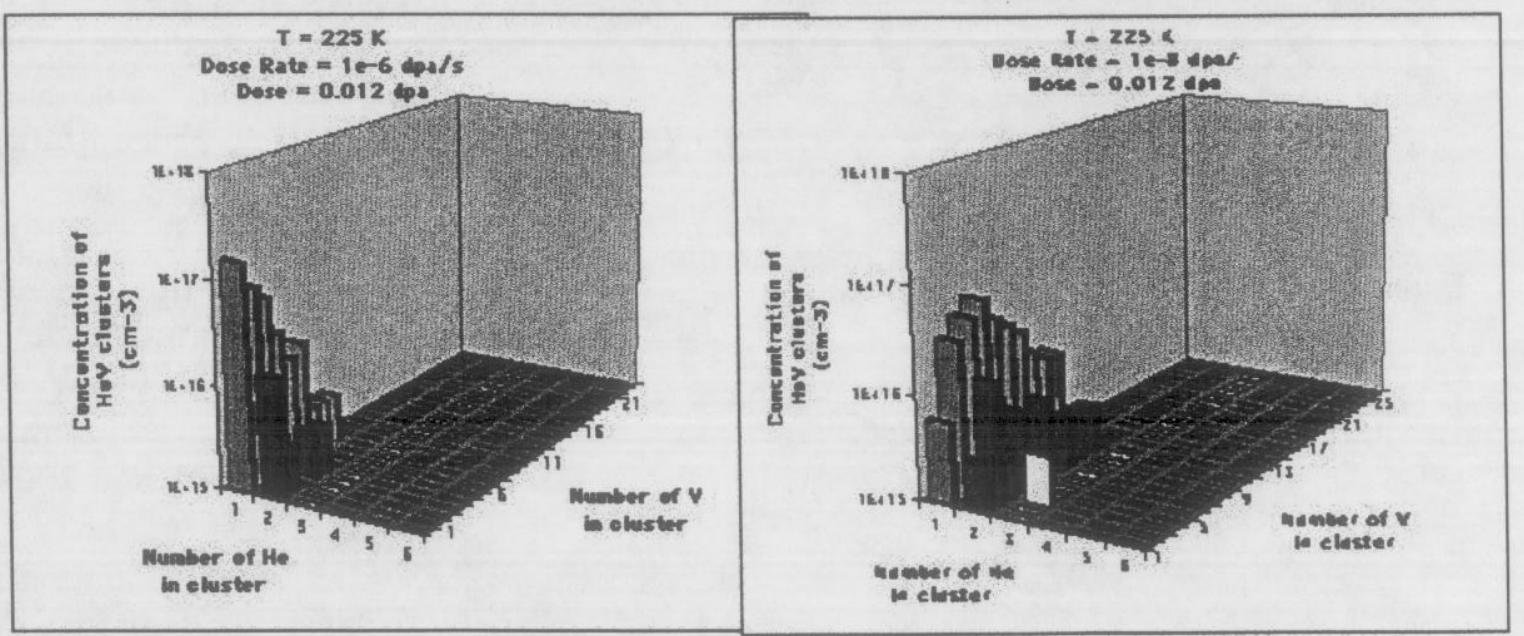

Figure 5. Nucleation of small yoids and bubbles as a function of dose rate at $T=225 \mathrm{~K}$. The figure shows that at the lower dose rate $\left(10^{-8} \mathrm{dpa} / \mathrm{s}\right)$ manymore HeV complexes nucleate and grow than at a higher dose rate of $10^{-6} \mathrm{dpa} / \mathrm{s}$. 
for two different dose rates, $10^{-6} \mathrm{dpa} / \mathrm{s}$ and $10^{-8} \mathrm{dpa} / \mathrm{s}$. Note that $10^{-6} \mathrm{dpa} / \mathrm{s}$ is a dose rate typical of neutron irradiation in a fast reactor environment. Clearly, a much larger number of $\mathrm{HeV}$ complexes is present at $10^{-8} \mathrm{dpa} / \mathrm{s}$ than at $10^{-6} \mathrm{dpa} / \mathrm{s}$. More significant is the fact that the distribution shifts towards larger, more stable clusters with larger $\mathrm{He} / \mathrm{V}$ ratios that exceed the critical size for growth. Note that at $10^{-6} \mathrm{dpa} / \mathrm{s}$ the distribution consists almost exclusively of clusters with one $\mathrm{He}$ atom and multiple vacancies, but at $10^{-8} \mathrm{dpa} / \mathrm{s}$ a shift toward more He per vacancy in the complexes takes place. The results clearly show that at the lower dose rates $\mathrm{HeV}$ complexes nucleate and start to grow very efficiently, and seem to indicate that the onset of void growth is at extremely low doses when the dose rate is very low.

Another consequence of low irradiation dose rate is the fact that peak temperature at which stable $\mathrm{HeV}$ complexes nucleate and grow beyond the critical size decreases. This is clearly illustrated in Fig. 6 which shows that while a high number of $\mathrm{HeV}$ complexes are present at $300 \mathrm{~K}$ for an irradiation flux of $10^{-6} \mathrm{dpa} / \mathrm{s}$, their density is much smaller at $10^{-8} \mathrm{dpa} / \mathrm{s}$. As a consequence, dimensional changes in this model system associated with $\mathrm{HeV}$ voids and bubbles disappear at temperatures around $300 \mathrm{~K}$, at least at the low doses simulated here.

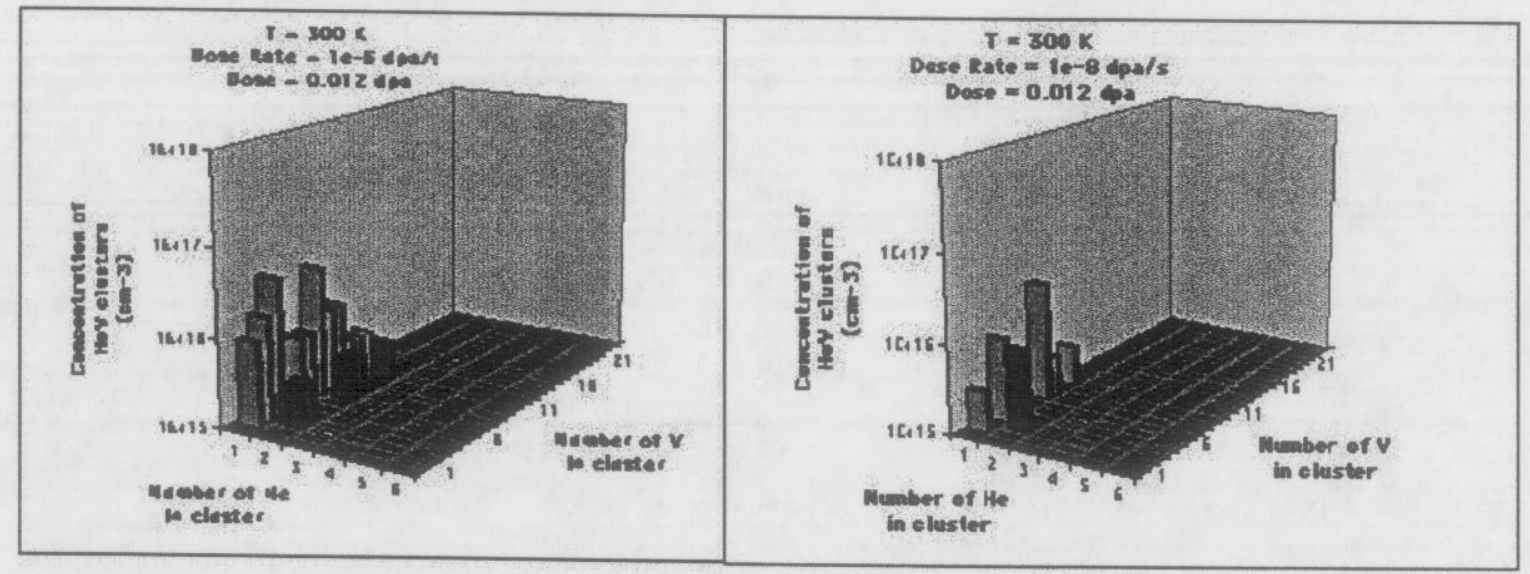

Figure 6. Distribution of $\mathrm{HeV}$ complex sizes at $300 \mathrm{~K}$ for two different dose rates, $10-6$ dpa/s and $10^{-8} \mathrm{dpa} / \mathrm{s}$. At the lower dose rate, small, subcritical HeV clusters dissolve before they can grow above a critical size indicating a shift in the temperature at which void growth can take place.

Another interesting class of defect that appears in these simulations is the cascadeinduced vacancy loop containing $\mathrm{He}$. These appear because He is a relatively fast diffuser and thus has a finite probability of finding a cascade remnant. He then serves to stabilize these loops and they dissociate at higher temperatures than do pure vacancy loops. The simulations show that at a given dose the concentration increases with temperature between $200 \mathrm{~K}$ and $300 \mathrm{~K}$ and then decreases to zero at $350 \mathrm{~K}$. One interesting question that has not yet been answered is whether addition of a He atom to a cascade-induced vacancy cluster prevents the collapse of the cluster to a loop or stacking fault tetrahedron resulting instead in a $3 \mathrm{D}$ void. If this were true, an increase in the rate of void production with dose concomitant with the number of $\mathrm{He}$ atoms arriving at cascade-induced vacancy clusters could be expected. Singh and Foreman [9] addressed this question some years 
ago and concluded that such a mechanism of void production was counter to experimental evidence. We have recently shown that the formation of an SFT from the cascade-induced vacancy remnant can occur in FCC metals in a timescale of a few piscoseconds even at $100 \mathrm{~K}$ indicating a very large driving force [10]. We thus conclude that formation of voids by the addition of $1 \mathrm{He}$ atom to a cascade-induced vacancy cluster is probably unfeasible.

As discussed in the introduction, one important question that simulations may help address is whether fast irradiation at high dose rates can be used to answer questions regarding system behavior at lower dose rates. The question that must be answered is what is the required temperature scaling that will ensure identical kinetic paths so that predictions can be made based on results at high dose rates. From the details of the simulations discussed above, we can obtain the rate of defect production as a function of temperature for different dose rates. One may then normalize these plots and determine whether the kinetics scale exactly with dose rate. If this is not the case, one may then determine what the temperature scaling required to reach the same microstructural state at different dose rates is. Figure 7 (a) and (b) shows the rate of $\mathrm{V}$ cluster and $\mathrm{HeV}$ cluster accumulation as a function of temperature at three different dose rates. In figures 7 (c) and (d) the same quantities are shown but normalized by the dose rate. As can be seen,
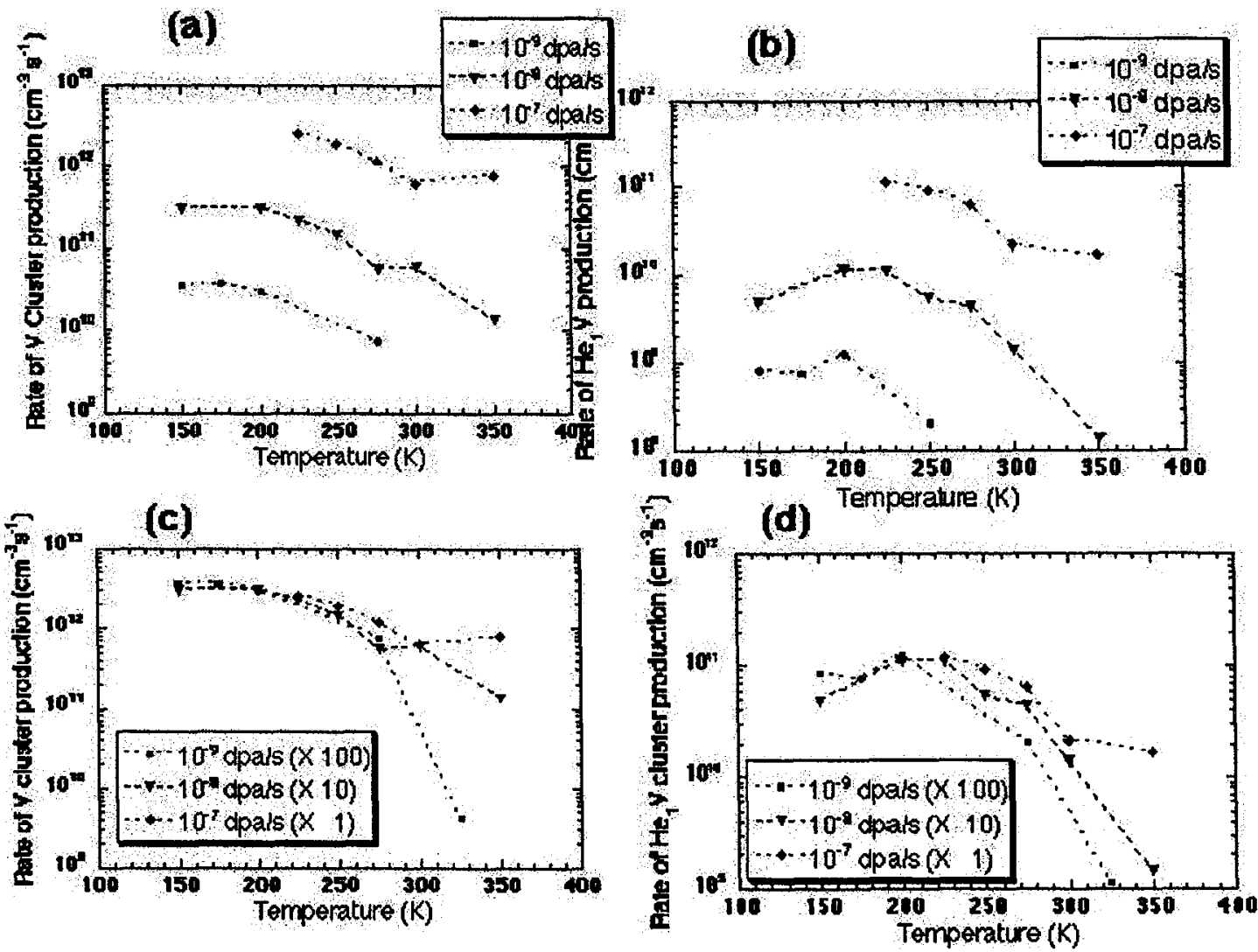

Figure 7. (a), (b) Rate of vacancy and HeV clusteraccumulation as a function of temperature for dose rates of $10^{-7}, 10^{-8}$, and $10^{-9} \mathrm{dpa} / \mathrm{s}$. (c), (d) same rates of damage accumulation but nomalized by the dose rate. Vacancy cluster accumulation scales well with the dose rate, but Hoy clusters do not. 
vacancy cluster accumulation obeys excellent kinetic scaling and the microstructure obtained after the same irradiation dose is independent of temperature and dose rate in this regime, particularly below $300 \mathrm{~K}$ where the damage accumulation is still linear at $0.03 \mathrm{dpa}$. Above $300 \mathrm{~K}$, vacancy clusters dissolve and the vacancies migrate to sinks such as I clusters, dislocations and grain boundaries. As a result, the damage density is so low at this dose that statistics are limited and the scaling in Fig $7 \mathrm{c}$ does not appear as good. For voids, Fig. 7d shows that the scaling with dose rate is not good for any temperature above $200 \mathrm{~K}$ where the vacancy becomes mobile. The reason for this difference is that vacancy clusters are produced directly in cascades and therefore their accumulation does not require diffusion. Accumulation follows first order kinetics, which are governed only by dissolution reactions and scale well with dose rate. In the case of $\mathrm{HeV}$ clusters on the other hand, diffusion is required for nucleation and growth, and therefore the kinetics are strongly influenced by temperature specially at temperatures above $200 \mathrm{~K}$ where the vacancy is mobile.

In figure 8 we show a plot of the $\mathrm{HeV}$ cluster concentration after scaling the

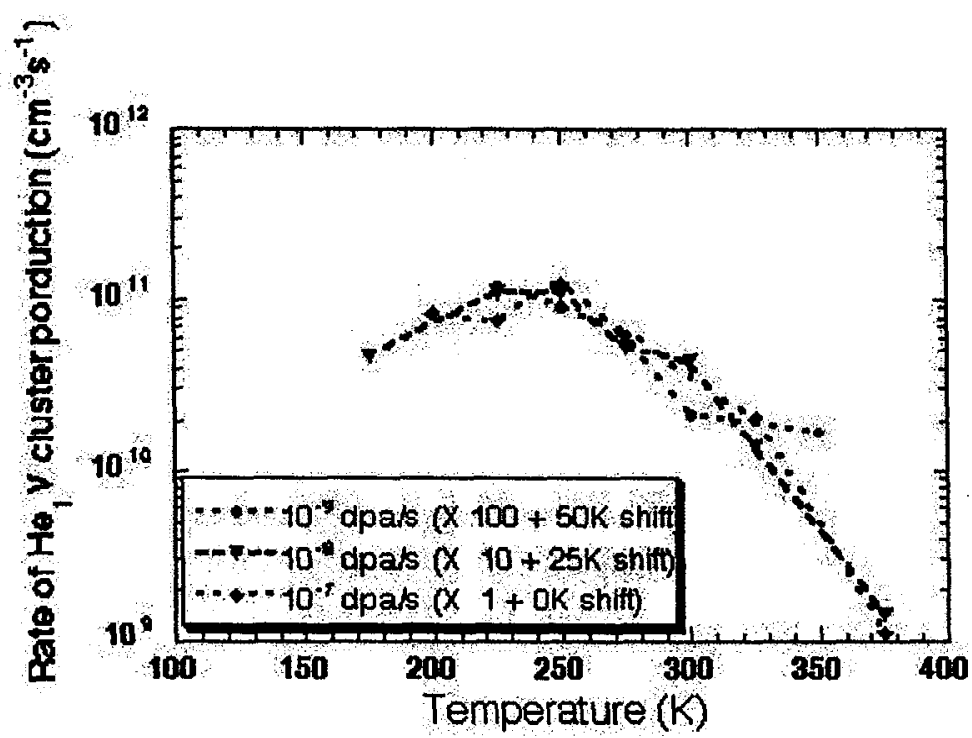

Figure e. Rate of HeY cluster production at several dose rates after scaling the results with a $25 \mathrm{~K}$ temperature shit per decade of decrease in dose rate.

of well designed accelerated aging experiments and robust multiscale modeling tools should provide a viable research avenue leading to predictions of materials aging.

\section{SUMMARY}

In summary, we have used MD and KMC simulations to investigate whether accelerated irradiation experiments can be used to investigate microstructure evolution in a model system that experiences radiation damage through undergoing alpha decay. We have described the kinetics of accumulation of $\mathrm{HeV}$ complexes as well as vacancy and interstitial clusters as a function of irradiation temperature, dose, and dose rate. We 
showed that at low temperature and flux nucleation of stable $\mathrm{HeV}$ complexes (voids and bubbles) takes place at extremely low doses. These may be thought of as precursors to the growth of larger voids and bubbles at higher doses. We also described the effect of temperature on the $\mathrm{HeV}$ complex size distribution and showed that growth beyond a critical nucleation size is not possible in this model system at low flux and temperatures above $300 \mathrm{~K}$. The results show that provided all other experimental variables are kept constant, a simple temperature shift scales the rate of nucleation and early accumulation of voids and bubbles as the dose rate is increased from $10^{-9} \mathrm{dpa} / \mathrm{s}$ to $10^{-7} \mathrm{dpa} / \mathrm{s}$. These simulation tools and results should help design and interpret the results of accelerated aging experiments.

Further work is underway to extend the applicability of these calculations to higher doses by including effects of cascade overlap, and to larger grain sizes. Such simulations should provide details of the dose rate and temperature dependence of defect cluster saturation as well as of the kinetics of void and bubble growth at higher doses.

\section{ACKNOWLEDGEMENTS}

This work was supported by ASCI and LDRD at LLNL. We have benefited from the support of and extensive discussions with W.G. Wolfer. Work performed under the auspices of the US Department of Energy by Lawrence Livermore National Laboratory under contract W-7405-Eng-48.

\section{REFERENCES - final version will follow}

[1] R.S. Averback and T. Diaz de la Rubia, Solid State Physics, Ed. By F. Spaepen and H. Ehrenreich, (Academic Press, New York, 1998) Vol 51.

[2] See e.g. H. L. Heinisch, Nucl. Inst. Meth. B102, 47 (1995).

[3] M.J. Caturla, N. Soneda, E. Alonso, B. D. Wirth, T. Diaz de la Rubia' and J.M. Perlado' J. Nucl. Mater. In press; UCRL-JC-

[4] Norgett, Robinson, and Torrens

[5] N. Soneda and T. Diaz de la Rubia, Phil. Mag. A78, 995 (1998); T. Diaz de la Rubia,

N. Soneda, M.J. Caturla, E. Alonso, J. Nucl. Mater. 251, 13 (1997).

[6] J. Adams and W.G. Wolfer

[7] M.J. caturla and B. Chan, unpublished.

[8]

[9] B.N. Singh and A. Foreman, J. Nucl. Mater.

[10] B. Wirth, V. Bulatov and T. Diaz de la Rubia (Unpublished) 1999. 